\title{
Computational approaches for predicting key transcription factors in targeted cell reprogramming (Review)
}

\author{
GUILLERMO-ISSAC GUERRERO-RAMIREZ, CESAR-MIGUEL VALDEZ-CORDOBA, \\ JOSE-FRANCISCO ISLAS-CISNEROS and VICTOR TREVINO
}

Tecnológico de Monterrey, Escuela de Medicina, Monterrey, Nuevo León 64710, México

Received September 26, 2017; Accepted February 27, 2018

DOI: $10.3892 / \mathrm{mmr} .2018 .9092$

\begin{abstract}
There is a need for specific cell types in regenerative medicine and biological research. Frequently, specific cell types may not be easily obtained or the quantity obtained is insufficient for study. Therefore, reprogramming by the direct conversion (transdifferentiation) or re-induction of induced pluripotent stem cells has been used to obtain cells expressing similar profiles to those of the desired types. Therefore, a specific cocktail of transcription factors (TFs) is required for induction. Nevertheless, identifying the correct combination of TFs is difficult. Although certain computational approaches have been proposed for this task, their methods are complex, and corresponding implementations are difficult to use and generalize for specific source or target cell types. In the present review four computational approaches that have been proposed to obtain likely TFs were compared and discussed. A simplified view of the computational complexity of these methods is provided that consists of three basic ideas: i) The definition of target and non-target cell types; ii) the estimation of candidate TFs; and iii) filtering candidates. This simplified view was validated by analyzing a well-documented cardiomyocyte differentiation. Subsequently, these reviewed methods were compared when applied to an unknown differentiation of corneal endothelial cells. The generated results may provide important insights for laboratory assays. Data and computer scripts that may assist with direct conversions in other cell types are also provided.
\end{abstract}

\section{Contents}

1. Introduction

2. Identifying key cell-state transcription factors

Correspondence to: Dr Victor Trevino, Tecnológico de Monterrey, Escuela de Medicina, 3000 Av Morones Prieto, Colonia Los Doctores, Monterrey, Nuevo León 64710, México

E-mail: vtrevino@itesm.mx

Key words: transcription factors, cell reprogramming, computational methods
3. Identification of pre-candidate TFs

4. Filtering the pre-candidate TF list

5. Finding key TFs in practice

6. Validation of target cell state TFs via different approaches in a well-known system

7. Estimation of target cell state TFs via different approaches in a novel system

8. Conclusions

\section{Introduction}

In tissue engineering and regenerative medicine, there is a need for large quantities of specific cell types $(1,2)$. For example, in corneal disease the use of transplants is essential, although access to corneal tissues is difficult given the shortage of tissue donors. Therefore, an alternative for generating specific corneal cells is needed (3). Furthermore, specific cell types are required in research for characterization, including studies on responses to treatment or genetic regulatory networks (4-7). For these needs, stem cell technologies hold the promise of providing a sufficient number of cells of specialized linages (2). Such promise is based certain factors, including the fact that cell differentiation may be reversed, that somatic cells may be induced to be pluripotent, or that cells may be forced to alter their identity or to transdifferentiate (8).

In this context, cell identity or cell state is thought to be a highly regulated process that depends on their epigenetic and transcriptional programming (9). The cell state is defined as the transcriptional output of a gene regulatory network (10). Thus, the cell state is principally controlled by the expression of transcription factors (TFs) forming specific network modules to ensure stable gene expression (7). However, genome analyses have identified approximately 2,000 TFs, and it is known that approximately one-half are expressed in a given cell (11). Thus, there is a requirement to elucidate which and how many TFs define specific cell states. The majority of the current literature in stem cells suggests that only a few TFs are required to maintain cell identity $(7,12-14)$. For example, only four TFs (MYC proto-oncogene bHLH transcription factor, Kruppel like factor 4, SRY-box 2 and POU class 5 homeobox 1) are required to maintain the pluripotency state $(8,15)$. These factors were identified from serial rounds of gene inclusion and withdrawal from a pool of 24 potential genes selected from studies performed on isolated 
genes. From this seminal work, other research groups identified several TFs for direct conversion (16-18). For example, glutamic-oxaloacetic acid transaminase 1 was used to convert fibroblasts into functional neurons (16) while GATA-binding protein 4 (GATA4), monocyte enhancer factor 2C (MEF2C) and T-box 5 (TBX5) were used to convert fibroblasts into cardiomyocytes (17). Moreover, alternative combinations of TFs may lead to very similar cell types (18), suggesting that redundancy exists in which the genetic regulatory networks characteristic of the cell identity may be established by similar or equivalent combinations of TFs.

Thus, if a cell state can be defined by a combination of TFs, in theory, any source cell type may be converted into any target cell type by establishing the expression of those TFs. Thus, if the differences in expression between the source and target cells are very small, one may consider subtle methods based on stimulating or blocking connected pathways. If the differences are large, as is commonly the case in converting fibroblasts to a lineage-distant cell type, one may opt to force expression by transdifferentiation or direct conversion (19-21) or via the generation of induced pluripotent stem cells (iPSCs) following the induction of the target cell type (13).

For other specific cell types, it is necessary to identify how candidate TFs may be obtained to begin with or how alternative TFs may be obtained. In the present study, the focus will be on providing simplified views of the computational approaches that have been proposed to identify a set or sets of putative TFs likely to control the cell state of the desired cell type. This proposed view may be highly illustrative for non-bioinformatics specialists for a number of reasons. Firstly, previously proposed computational methods are complex. Secondly, the literature accompanying the computational methods is highly technical. Thirdly, the descriptions of certain methods may appear vague for non-specialists. Fourthly, certain data (specifically, the networks) or computer scripts and tools described in the algorithms are currently unavailable, complicating re-implementations. Finally, the majority of approaches were proposed using ad-hoc parameters and specific datasets. In addition, for bioinformatics specialists, a succinct starting point for novel implementations was provided by the present review. To overcome the aforementioned difficulties, a simplified and unified view of current methods was provided, which may be summarized thus: i) The establishment of the population of cell types; ii) the estimation of candidate TFs from cell populations; and iii) the filtering of TF pre-candidates (the most challenging element). Derived from these summarized concepts, clues as to how the methods work are provided, in addition to knowledge as to how to overcome or approach difficulties. Possible ways in which these computational methods may be re-implemented and adapted to provide a preliminary list of TFs are additionally provided.

\section{Identifying key cell-state transcription factors}

The idea that cell states are associated with the binary decision of cell fates has long been proposed (22). However, computational approaches to identify key TFs governing cell states are more recent. In practice, an aim may be to directly convert a specific source cell type into a target cell type; therefore, the most important component is the estimation of the target cell state, since the state of the source cell type may be forced to change. The source cell type is important to be able to estimate those TFs that may be redundant and perhaps do not required manipulation; this may be easily performed by comparing expression levels. Therefore, the majority of methods primarily focus on the estimation of TFs controlling the target cell state. The following sections consider the approaches of recent studies (23-26), which are accordingly referred to as Cahan et al (23), D'Alessio et al (24), Rackham et al (25) and Okawa et al (26).

Identification of TFs via differential expression. Under the assumption that the cell identity is controlled by the gene expression level of a specific set of TFs, it follows that the identity of cell types be controlled by either different levels of the same set of TFs or a different set of TFs (7). In any case, the same operation is needed: The identification of the characteristic and distinct gene expression levels. This is best known as differential expression. Since this operation involves the comparison between at least two populations assumed to be distinct, the target cell type population and the 'background' population require careful selection. In theory, if these populations are well defined and the available data are highly representative and precise, it ought to be possible to create a small list of TFs. However, even today, the available data are scarce, highly noisy and contaminated with different populations of cells; the data from in vitro assays may not reflect genuine in vivo properties; and the computational and statistical tools may be imperfect. Therefore, the output of the differential expression between the defined cell populations usually generates large lists of pre-candidate TFs.

Filtering problem. Assuming that the number of TFs controlling the cell identity is small, this large list of pre-candidate TFs ought to be highly contaminated with false-positive calls representing cell-state-irrelevant TFs that require filtering out. Although certain irrelevant TFs may be easily identified by expert researchers and available biological knowledge in the literature, this process is time-consuming and may be prone to misinterpretations, errors and omissions. In addition, certain TFs may not be well studied or studied at all. Furthermore, manual filtering of the list causes difficulties in the scoring or ranking of TFs according to the scientific literature. Therefore, the systematic filtering and ranking of pre-candidate TFs is a challenging issue. This filtering process is obscured in original research articles due to the complexity of their implementations. The majority of the considered methods perform this filtering procedure analyzing the TFs within the context of biological networks. Although this may be considered to be a drawback by non-bioinformatics specialists, this step need not be very complicated to help to reduce large lists. In particular, within the examples provided, even when no filtering is used, sensible results may be obtained if target and non-target cell populations are well defined.

In summary, the proposed view of the process to identify TFs likely controlling a cell state is demonstrated in Fig. 1 and is discussed in the following sections. In practice, it may be advisable to start with a specific source cell type for induction to a target cell type, whereas the majority of methods focus on the target cell type to identify TFs associated with the cell 
A Definition of cell populations Available cell types

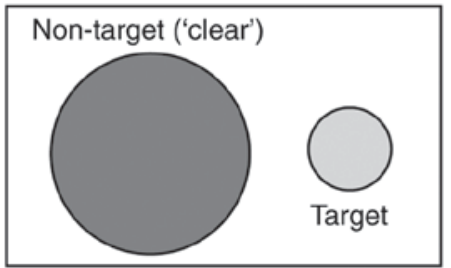

B Differential transcription factors

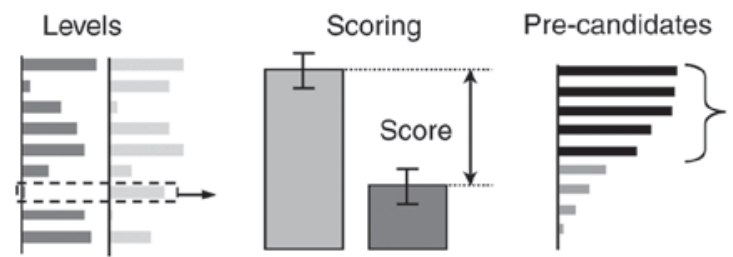

C Transcription factors filtering
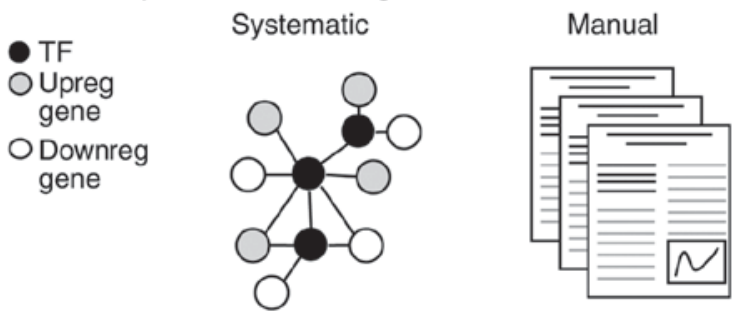

Figure 1. Simplified view of TF identification for cell conversion. (A) Process of defining at least two cell populations. (B) Differential expression analysis of TFs between defined populations to identify pre-candidate TFs. (C) Filtering process of pre-candidates in order to generate a short list of TFs whose overexpression will likely control the desired cell state. TF, transcription factor.

state (23-25). Thus, once the cell types have been identified, as depicted in Fig. 1, the TF expression profile of the source cell type is compared with the target to identify those TFs required to induce from that particular source.

Defining the populations of cell types. The first step consists of defining at least two populations of cell types (Fig. 1A), which are referred to as target and non-target cell types. A comparison of conceptual definitions by the authors is demonstrated in Fig. 2 and discussed in the following paragraphs.

Datasets used. Gene expression data are required to be uniformly annotated for target and non-target cell types. Therefore, the majority of methods utilize information from the vast collections of microarray gene expression data available from the Gene Expression Omnibus (GEO) $(27,28)$ and ArrayExpress $(29,30)$, or from more recent next-generation sequence repositories in ENCODE (31) or FANTOM (6). The repositories used are detailed in Table I. The majority of the studies discussed in the present review used GEO microarray data, except Rackham et al (25), who used FANTOM5. They studied human data, although Cahan et al (23) additionally included murine data. The majority of the studies included numerous cell types; however, Okawa et al (26) used progenitor and daughter cell types from specific third-party authors.

\section{Cell populations}

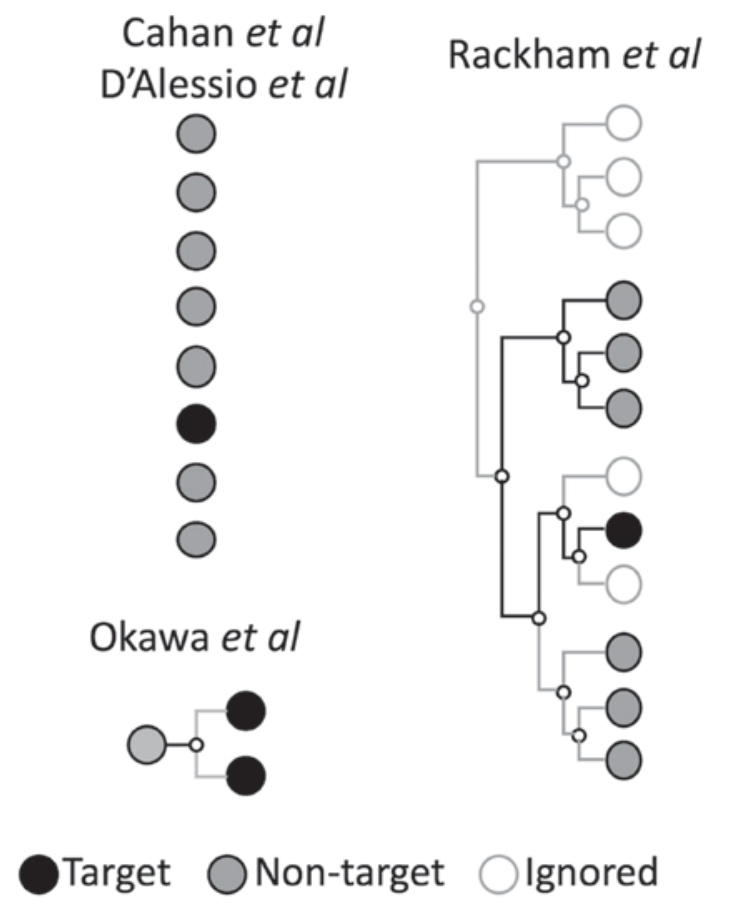

Figure 2. Comparison of the definition of cell populations.

Target cell type. For the target population, which is generally the easiest to delimitate, a number of considerations are noteworthy. First, the target cell type is required to be well represented. From the authors reviewed herein, various experiments were performed in vitro, while others have been obtained from tissue samples. The experiments performed in vitro have the advantage of a well-defined cell type, whilst the tissue samples may represent a mixture of distinct cell types generating an average cell state that may not properly represent the desired target. Second, the gene expression data may reflect the cell state of an individual donor instead of a population-generalizable cell state. Thus, it is desirable to include as many individuals as possible. Third, repetition is desirable as gene expression data are noisy, which is worsened by the technology used to acquire the data (particularly microarrays). In summary, the targets used for each method are mentioned in Table I. Rackham et al (25) used a hierarchical ontology definition of cell types from FANTOM5 to define a particular target cell type; they ignored closely associated cell types (Fig. 2). In this way, they favored the purity of the cell state. However, they lost generality as closed cell types may help to eliminate non-specific TFs, leading to larger lists of pre-candidate TFs if their implementation is not followed thoroughly. On the contrary, Okawa et al (26) used a specific cell type contrasted with the closest associated cell types (daughter cell types; Fig. 2). This has a number of advantages since the comparison of close, although distinct, cell types may lead to the clear identification of controlling TFs. However, this method is unable to be generalized as the type of experimental setting (well-defined progenitor and daughter cell types) required to run this approach is not as common in the data repositories and must be performed in advance to generate the data. D'Alessio et al (24) and Cahan et al (23) first defined a 
Table I. Definition of populations of cell types by all methods.

\begin{tabular}{|c|c|c|c|c|}
\hline Author, year & Data & Target & Non-targets & (Refs.) \\
\hline Cahan et al, 2014 & $\begin{array}{l}\text { GEO, queried datasets, } \\
16-20 \text { cell types }\end{array}$ & $\begin{array}{l}\text { Several samples of the same } \\
\text { cell or tissue type }\end{array}$ & Remaining cell types & (23) \\
\hline D'Alessio et al, 2015 & $\begin{array}{l}\text { GEO, } 504 \text { datasets, } \\
233 \text { cell types }\end{array}$ & $\begin{array}{l}\text { Several samples of the same } \\
\text { cell or tissue type }\end{array}$ & $\begin{array}{l}\text { Remaining cell types } \\
\text { (balanced) }\end{array}$ & (24) \\
\hline Rackham et al, 2016 & $\begin{array}{l}\text { FANTOM5, >700 } \\
\text { datasets (CAGE-Seq) }\end{array}$ & Samples of the same cell type & $\begin{array}{l}\text { Remaining cell types but } \\
\text { avoiding close and distant } \\
\text { related ones }\end{array}$ & $(25)$ \\
\hline Okawa et al, 2016 & GEO, Specific data & A daughter cell type & $\begin{array}{l}\text { The progenitor and sister } \\
\text { cell types }\end{array}$ & (26) \\
\hline
\end{tabular}

GEO, gene expression omnibus.

number of classes of tissues or cell types and compared each class against the remaining classes (Fig. 2). In each class, they used numerous samples, avoiding individual and noise effects.

Non-target cell types. Following removal of the target data, the non-target data are commonly obtained from the remaining tissue or cell types of the defined datasets (Table I). Nevertheless, Rackham et al (25) removed distantly related samples, probably due to a highly-curated cell lineage ontology. This has the advantage of removing false differentially expressed TFs that may control specialized functions in distant and target cell types, presumably via an upregulated TF. Nevertheless, this concept is only useful if the TF differential scoring depends on downregulated TFs, as in Rackham et al (25). Therefore, the removal of distant cell types may be redundant if only upregulated TFs are considered and there are no large combinational effects in TFs. In addition, the threshold required to determine distance is hard to define, complicating further tests in diverse scenarios. In Okawa et al (26), the target was one of the daughter cell types, and therefore the non-target was formed by the progenitor and the sister cell type. An issue with using large collections of samples in the non-target is that it may be highly disproportional to the number of samples. To avoid this overrepresentation, in D'Alessio et al (24), the non-target dataset was balanced by selecting a representative sample from the collection of samples of each cell type.

\section{Identification of pre-candidate TFs}

The four computational methods proposed used different approaches, which are conceptually summarized in Fig. 3. Theoretically, however, the identification of putative TFs may be obtained by identifying TFs whose expression is statistically different. Therefore, parametric, non-parametric or permutation tests may provide similar results (32-34). Statistical tests provide a P-value that is useful, although it does not represent the magnitude of the difference between two average expression levels and is sensitive to the variance and number of samples (35). Alternatively, these issues may be solved by using combinations of the P-value and fold-change, for example in Rackham et al (25), where the score per TF

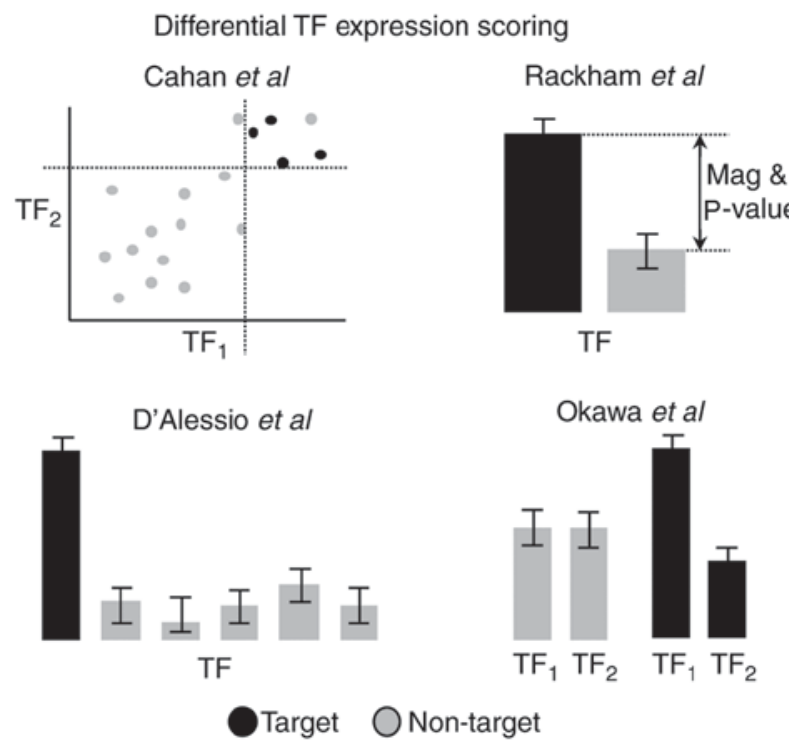

Figure 3. Comparison of conceptual definitions to identify TF differences. $\mathrm{TF}$, transcription factor; mag, magnitude.

is based on the absolute magnitude of the fold-change multiplied by the (negative) logarithm of the P-value. Nevertheless, certain of the methods reviewed demonstrate a preference for other strategies (Table II). For example, D'Alessio et al (24) used Jensen-Shannon Divergence (JSD), which is a measure of the discrepancy between distributions. JSD was used to score differences between the observed TF expression profiles and idealized ones. These idealized profiles are formed by combining high expression in the corresponding target cell type and no expression in the remaining cell types.

Instead of comparing one TF across cell types, Okawa et al (26) and Cahan et al (23) compared pairs of TFs (Table II and Fig. 3). The comparison of pairs is based on the concept that balanced expression between two TFs is associated with cell identity (36-38). Okawa et al (26) proposed the normalized ratio difference (NRD) to score all pairs of TFs that are similarly expressed in a progenitor cell type, and highly different in and between daughter cell types. Cahan et al (23) additionally compared pairs of TFs, using the metric of the 
Table II. Identification of differential expressed TF.

\begin{tabular}{lll}
\hline Author, year & \multicolumn{1}{c}{ Method } & \multicolumn{1}{c}{ Comparison } \\
\hline Cahan et al, 2014 & Tissue-Specific Context Likelihood of Relatedness & Pairs of co-expressed TF \\
D'Alessio et al, 2015 & Jensen-Shannon Divergence & Per TF \\
Rackham et al, 2016 & Combines P-values and fold-change & Per TF \\
Okawa et al, 2016 & Normalized Ratio Difference & Pairs of swap-expressed TF
\end{tabular}

$\mathrm{TF}$, transcription factor.

context likelihood of relatedness (CLR). The CLR is a measure that favors TFs that are highly correlated (by mutual information) and whose correlations are within the top ranked to increase the probability of genuine associations (39). Notably, while Okawa et al (26) favored pairs of TFs whose expression was different in daughter cells, Cahan et al (23) favored TFs that were co-expressed and whose expression levels were cell-type specific (as explained in more detail in the following section). These opposing views are associated with the input data: Okawa et al (26) used cell types that were extremely close in the lineage, whereas Cahan et al (23) use tissue types that are more distant (Fig. 2). By definition, these methods will generate much larger lists of pre-candidates compared with those comparing one TF at the time. For example, assuming that there are $2,000 \mathrm{TFs}$, there would be $1,999.000$ pairwise comparisons vs. 2,000 when only one TF is assessed at the time. Thus, these methods require extensive filtering.

\section{Filtering the pre-candidate TF list}

The objective of this step is to further filter the pre-candidate list to end up with a short list of candidate TFs whose overexpression will likely control the desired cell state. This step is frequently the most complex and time-consuming; it depends on the length of the pre-candidate TF list and the rules defined in the filters. In assays of one TF, including in D'Alessio et al (24) and Rackham et al (25), if 5\% of the 1,000 expressed TFs are differential, a list of $\sim 50$ TFs is expected. This estimate is not far from reality, supposing that few TFs control the cell state by means of regulating further TFs, which thus regulate the downstream effector genes. Furthermore, for methods that compare pairs of TF, including in Okawa et al (26) and Cahan et al (23), and even optimistically estimating that only $0.1 \%$ of pairs are of interest, $\sim 1,000$ pairs of TFs would have to be analyzed from the $\sim 1,000.000$ TF pairs generated. Unless a shorter pre-candidate list is obtained, analyzing the TF list manually by reading scientific literature or browsing databases by hand may be arduous, prone to errors and time-consuming. Therefore, the filtering procedures proposed are focused on setting sensible rules that are approachable with current databases.

Thus far, the focus has been on differential TFs; however, other non-TF genes require consideration. They are involved in signal propagation or provide cell type-specific functions and should also be considered. Therefore, to completely explain the observations, the rules must be based on maximizing the control over all observed differentially expressed genes (DEGs), irrespective of the gene function (TF or not). Thus, the rules may be easily stated as 'show all TFs directly or indirectly controlling all DEGs.' If all regulatory associations between TFs and other types of genes are known, this statement may be more easily implemented compared with the current methods. Nevertheless, the current databases are far from being complete, are context-specific (by culture or tissue) and are likely to include errors. Therefore, in the following paragraphs, how these rules were implemented in each method is explained and an overview is illustrated in Fig. 4. The majority of the methods make use of networks, databases and other tools to integrate information and connect the TFs with themselves and with other DEGs.

In Cahan et al (23), a genetic regulatory network was built upon the significantly correlated pairs of genes using the CLR. As these networks are frequently large, the InfoMap tool was used to split this large network into smaller, highly connected sub-networks (40). Furthermore, each sub-network was evaluated using gene set enrichment analysis (GSEA) (41). GSEA generates a score depending on the position of the genes in the sub-network relative to all genes. If the genes are randomly distributed, the GSEA score is low, whilst if the expression levels are more concentrated in closer positions, the GSEA score increases. If the GSEA score of a sub-network obtained from tissue $\mathrm{A}$ is higher compared with other tissues, this sub-network is defined as specific for tissue A. Subsequent to executing this procedure in all sub-networks present in all tissues, Cahan et al (23) ended up with 76 tissue-specific sub-networks. Thus, on average, approximately five sub-networks were expected in each of the 15-20 tissues or cell types. From this, the study aimed to identify which sub-networks and which genes within the sub-networks were more likely to be manipulated, starting from a source cell type. To evaluate the former, the expression of each gene within the target cell type sub-network was compared against that of the source cell type; if the expression levels were similar, no larger alterations were required, whilst if the expression levels were very different, the sub-network had to be re-established and was therefore a target for manipulation. To assess the genes within the sub-networks, a network influence score (NIS) was estimated. This NIS depends on the difference in TF expression between the source and the target, the differences in the expression of the predicted genes regulated by that TF, and the number of regulated genes. In brief, a large network was split into sub-networks, filtered for tissue specificity, further 
Transcription factor filtering
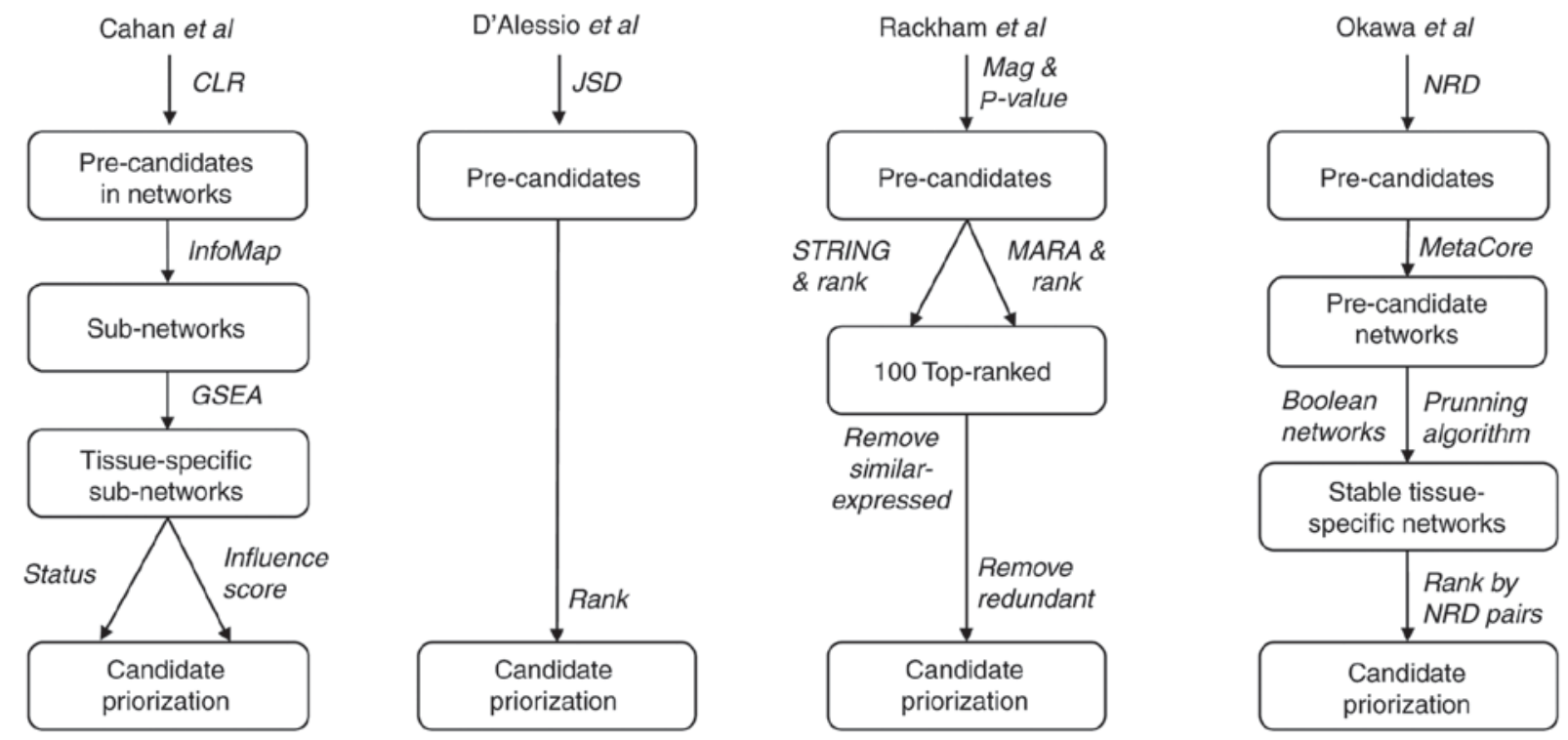

Figure 4. Comparison of the generation of candidate TFs. TF, transcription factor; CLR, context likelihood of relatedness; JSD, Jensen-Shannon divergence; NRD, normalized ratio difference; GSEA, gene set enrichment analysis.

filtered to detect those expressed at different levels and, finally, TFs were ranked within the resultant sub-networks. Cahan et al (23) demonstrated acceptable predictions in a number of conversion systems and suggested that direct conversions are less similar to the in vivo tissues compared with those conversions obtained from iPSCs.

Elsewhere, D'Alessio et al (24) used the JSD metric against an idealized profile to evaluate each TF between the target and non-target cell types in around 233 cell types. This procedure yielded 503 TFs across these cell types. A total of $\sim 60 \%$ of the TFs were considered to be pre-candidates in fewer than four cell types, demonstrating that most were cell-type specific. From the experiments, the study of D'Alessio et al (24). focused on the top 10 TFs for induction. This approach was validated by comparing their predictions to well-known conversion systems, including iPSCs, neural precursor cells, cardiomyocytes, hepatocytes, motor neurons, pancreatic islets cells and melanocytes. Furthermore, D'Alessio et al (24) predicted and experimentally validated their approach in the conversion of fibroblasts to retinal pigment epithelial-like cells.

In Rackham et al (25), the pre-candidate list of TFs was generated using a combination of a tissue-specific P-value and the magnitude of the difference. For the filtering, two additional network influence scores were used for each TF, which were estimated from MARA (42) and STRING (43) networks. These network scores depend on how many genes are connected to each TF, how far the connection is (number of nodes), and the score of the regulated gene (P-value and magnitude). Subsequently, the ranks of these three scores were added and ranked to provide a final rank. The first filter consisted of using only the TF within the top 100 final ranks. The second filter removed the TFs that were expressed in the source and target cell types. The third filter removed those redundant TFs that shared the majority of their targets with other TFs regulating more genes. A fourth filter was applied to include the top eight TFs. The approach was validated in at least five systems, involving conversions from fibroblasts to iPSCs, myoblasts, hepatocytes and cardiac cells, and from $\mathrm{B}$ cells to macrophages. Finally, two novel conversions were predicted and tested experimentally, converting fibroblasts to keratinocytes and keratinocytes to microvascular endothelial cells.

Okawa et al (26) used the NRD metric to evaluate and select pairs of TFs. Subsequently, the MetaCore network database (44) was used to first filter TFs with over seven connections. This was based on the observation that important TFs are highly connected in MetaCore. The next filter removed unnecessary nodes of the network, based on the assumption that a cell type may be stabilized by a gene regulatory network that was additionally stable in the two daughter cell types. For this, the study re-implemented a sub-network-finding optimization algorithm combined with Boolean networks (45). A Boolean network is a methodology that is able to identify attractor states (46). These attractors were interpreted in biological cells as stable states that may be compared with the states of daughter cell types filtering those matching sub-networks. Subsequent to running the algorithm numerous times, the following filter looked for all sub-network solutions that contained at least one upregulated TF. Subsequently, the sub-networks were ranked based on the number of NRD pairs present, NRD pairs directly connected and lesser regulatory connections. This approach was validated in five stem cell systems, including mouse embryonic stem cells, mouse and human hematopoietic stem cells, mouse neural stem cells and mouse mesenchymal stem cells. Furthermore, the induction of neuronal and astrocyte differentiation was predicted and experimentally confirmed in a mouse neuronal stem cell system.

\section{Finding key TFs in practice}

In this section, the focus is on how to estimate the key TFs for the target cell type of interest in an easy and practical way, 
Table III. Resources available for finding key TF.

Author, year

Resources and limitations

(Refs.)

Cahan et al, 2014

CellNet: Web interface and R package. Any source cell type as input but only from certain Affymetrix arrays, and Illumina arrays (in R). Only specific target cell types are available

D'Alessio et al, 2015 File for 233 cell type predictions. Manual estimations are possible for a target. Source is not used.

Rackham et al,2016 Mogrify: Web interface. Specific for several already cataloged source and target cell types.

Okawa et al, 2016 None available.

$\mathrm{TF}$, transcription factor.

while commenting on each approach. Ideally, the prediction would be made to manipulate a source cell type to achieve a target cell type. However, the majority of methods are restricted to specific sources, targets, or both. A summary is provided in Table III, and details are provided in the following paragraphs. An estimation of gene expression values was assumed and their annotation for the target cell type was available either from microarrays or from RNA-Seq. An overview of the available tools is provided followed by a practical example.

Overview of available tools. Cahan et al (23) provided a web interface (cellnet.hms.harvard.edu) and an $\mathrm{R}$ package (pcahan1.github.io/cellnetr) termed CellNet, which may be used to feed gene expression data of the source cell type or the already manipulated cell types. The output was composed of three main sections. The first output was a classification of input samples into cell types used in CellNet. The second output demonstrated how well each cell type-specific genetic regulatory network was established across the input samples. This helped to identify the networks that were required to be manipulated to achieve a cell type. The third output demonstrated the TFs having larger differences within networks, indicating which TFs required manipulation. For the web version, only Affymetrix (Thermo Fisher Scientific, Inc., Waltham, MA, USA) microarrays were able to be used. For the R package version, Illumina (Illumina, Inc., San Diego, CA, USA) microarray data were additionally able to be used.

For D'Alessio et al (24), if the target cell type was already in the list of the 233 cell types processed [available in the supplementary information of the study (Table SI)], the top-ranked TFs demonstrated were able to be used ( 10). If the cell type was not demonstrated, and to avoid reconstructing the entire study, the JSD or JSD-like value for the target cell type was estimated. Spreadsheet software using the predictions available for the 233 cell types was used. First, the TF expression of the target cell type was required to provide a rank of expression. Second, for each TF, the number of times this TF was counted in the top 10 other cell types was obtained. Third, for each TF, the minimum rank of the TF in all other cell types was obtained. Fourth, scatter plots of the target rank of TFs against those in the second and third steps was displayed. Fifth, TFs that were top ranked in the target and had low counts in the first scatter plot and/or were top ranked in the target and had higher ranks in the second scatter plot were estimated. These steps attempted to provide an easy approximation of the process followed by D'Alessio et al (24) instead of an accurate calculation, although these steps may be used as an easy starting point.

From Rackham et al (25), a web interface is available (www.mogrify.net) in which the source and target cell types were specified from those already considered. The top eight ranked TFs were elucidated in a few seconds. Unfortunately, to estimate the possible TFs for a non-listed cell type, it is necessary to reconstruct the study of Rackham et al (25) since no datasets are provided.

Notably, in Okawa et al (26), neither implementation nor supplementary information was available. Thus, it is necessary to reconstruct the study to make predictions using this method. The MetaCore regulatory network used is not currently available, thus a different network database or another method for estimation may be used. Therefore, putative results may be different.

\section{Validation of target cell state TFs via different approaches in a well-known system}

To demonstrate that this simplified view was able to generate sensible TFs, the first two concepts were applied in a well-known system, transdifferentiation towards cardiomyocytes $(\mathrm{CM})$.

Target and non-target datasets. The target CM data were obtained from the GEO/NCBI, with accession no. GSE45878, for the 62 samples annotated as 'Heart.' The dataset consists of 837 samples from diverse tissues. The non-target dataset was obtained from the remaining 775 samples and the number of probes was 22,704 .

Data pre-processing. The two datasets were quantile normalized and scaled to a uniform distribution between 0 and 1, representing no expression and maximum expression. To recognize TFs, 'transcription' and 'factor' in the annotated description were used. Additionally, AnimalTFDB was used for the TF annotation (47). Thus, 1,392 TFs were considered. The target and the non-target datasets, in addition to tissue annotation, are available at bioinformatica.mty.itesm. $\mathrm{mx} / \mathrm{CEC}-\mathrm{TF}-\mathrm{Example}$. 
Score implementations. A total of five scores were used, two taken from basic concepts of differential expression, and three inspired by those scores used by the methods reviewed here. 'Delta' is the difference in mean expression values between the target and non-target cell types. 't-test' is the P-value of the unequal variance t-test applied to target and non-target cell types. 'Rackham' is -Log10(p-t-test)xIDeltal, as in Rackham et al (25). 'D'Alessio' is the sum of 100 JSD scores between the observed and the ideal profile. The observed profile was estimated using the average target expression together with $\mathrm{k}=3$ random samples from non-targets (increasing values of $\mathrm{k}$ did not increase similarity to other scores). This process was similar, although not identical, to that implemented in D'Alessio et al (24) (details of the algorithm in the supplementary information were not clear). 'Okawa' was an adaptation of the Okawa et al (26) metric to cell types different from progenitor-daughter. It was estimated $\left(\mathrm{TF}_{\mathrm{Ti}}-\mathrm{TF}_{\mathrm{Tk}}\right)-\left(\mathrm{TF}_{\mathrm{Ni}}-\mathrm{TF}_{\mathrm{Nk}}\right)$, where $\mathrm{T}$ and $\mathrm{N}$ sub-indexes refer to the mean expression values of the target and non-target cell types, respectively, $\mathrm{i}$ is a particular TF, and $\mathrm{k}$ represents all TFs. This metric generated very similar results to the NRD (which involves ratios that are more unstable, although the script provided includes the NRD estimation). To generate a single score per TF, the number of times a TF was included in differences between the top $1 \%$ of pairs was counted. The score of Cahan et al (23) was not implemented since the tissue specificity is reached following large operations in networks (the scripts and data are available at bioinformatica.mty.itesm.mx/CEC-TF-Example).

Summary of the results. Table IV illustrates the results of the top 20 genes generated by the five scoring methods. The table summarizes the most frequently mentioned TFs and previously reported experimental findings. All of the top seven TFs listed have already been used experimentally for the conversion of different cell types to cardiomyocytes, including T-box 20 (48,49), GATA4, TBX5 (50-52), NK2 homeobox 5, and heart and neural crest derivatives expressed $2(50,52)$. However, a widely used TF in this conversion, $M E F 2 C(17,50,51,53)$, was not present in the list. Following revision, this gene was not marked as a TF in the present databases. Even if $M E F 2 C$ was added as a TF, it was not included in the top 20 of any scoring method. This TF appears to be important as its overexpression removal did not generate cells expressing important cardiac markers (17). A recent meta-analysis specific for CM differentiation did not identify $M E F 2 C$, although it did identify a family gene, $M E F 2 A$ (54). Although this result may give some clues regarding $M E F 2 C$, it is difficult to conclude the extent of its importance from this data alone. On the other hand, this example demonstrates that the majority of TFs may be obtained via straightforward application of simple concepts, as depicted in detail for the top 20 TFs identified in Table IV (55-61), but also highlights that is possible that not all factors required are obtained with the current methods.

\section{Estimation of target cell state TFs via different approaches in a novel system}

To provide a practical and simple way to reproduce an example and a comparison of different approaches as a starting point, corneal endothelial cells (CEC) were used as a target cell type. The tools and data available (Mogrify, CellNet and the D'Alessio et al supplementary information) did not include CEC and therefore were not used. As the datasets represented in these tools are limited, this example represents a likely scenario for specific cell types. Re-implemented scores inspired by the revised methods and the pre-candidate lists are compared. This demonstrates that the first two steps are highly useful and relatively easy to implement. Subsequently, data are processed in $\mathrm{R}$ (cran.r-project.org). The scripts and the data required to reproduce the results are available in bioinformatica.mty.itesm.mx/CEC-TF-Example.

Target and non-target datasets. The target CEC data were obtained from GEO/NCBI with accession no. GSE58315 (62). The dataset consisted of 11 corneal endothelial cell samples from adults, adolescents and preschoolers. The non-target dataset was obtained from a preliminary study on gene co-expression networks (63). This dataset consisted of 445 samples representing $>136$ tissues from the two most popular Affymetrix platforms (HG-U133) extracted from the GEO/NCBI. The number of probes was $>50,000$; however, due to the different versions of Affymetrix microarrays, certain samples provided data for only 22,000 probes.

Data pre-processing. The two datasets were quantile normalized and scaled to a uniform distribution between 0 and 1 , representing no expression and maximum expression. For the non-target dataset, the JetSet package was used to identify a representative probe for each gene (64). To recognize TFs, the Affymetrix annotation of the platform GPL570 was used to look for 'transcription' and 'factor' in the annotated description. Additionally, the TFs annotated in AnimalTFDB were used (47). Thus, 1,478 TFs were considered. The target and the non-target datasets along with tissue annotation are available in bioinformatica.mty.itesm.mx/CEC-TF-Example. For the target dataset and duplicated probes per gene, the probe whose standard deviation was highest was selected. Only the 16,098 genes matching in the two datasets (by gene symbol) were used, of which 1,408 were annotated as TFs.

Score implementations. A total of five scores were used, as demonstrated in the aforementioned cardiomyocyte analysis.

Comparison of resultant scores. Whether the re-implemented scores were similar to each other was investigated. Fig. 5A illustrates the results for the 1,408 annotated TFs. It is clear that Delta, a measure of differences in the averages between target and non-target expression, correlated with all other scores. Rackham, as expected, was associated with t-test (Delta and t-test are part of the calculation). D'Alessio was negatively correlated with Delta, although highly variable (lower D'Alessio scores tended to be similar to high Delta scores). The Okawa score seemed to be a proxy of Delta irrespective of the sign. Overall, these results suggested that the scores are associated with differential expression, supporting the summarized view.

Comparison of the generated TF list of pre-candidates. To demonstrate an overview of the top selected genes per score, the TF identity of the top 20 TFs was investigated (Fig. 5B). It is 


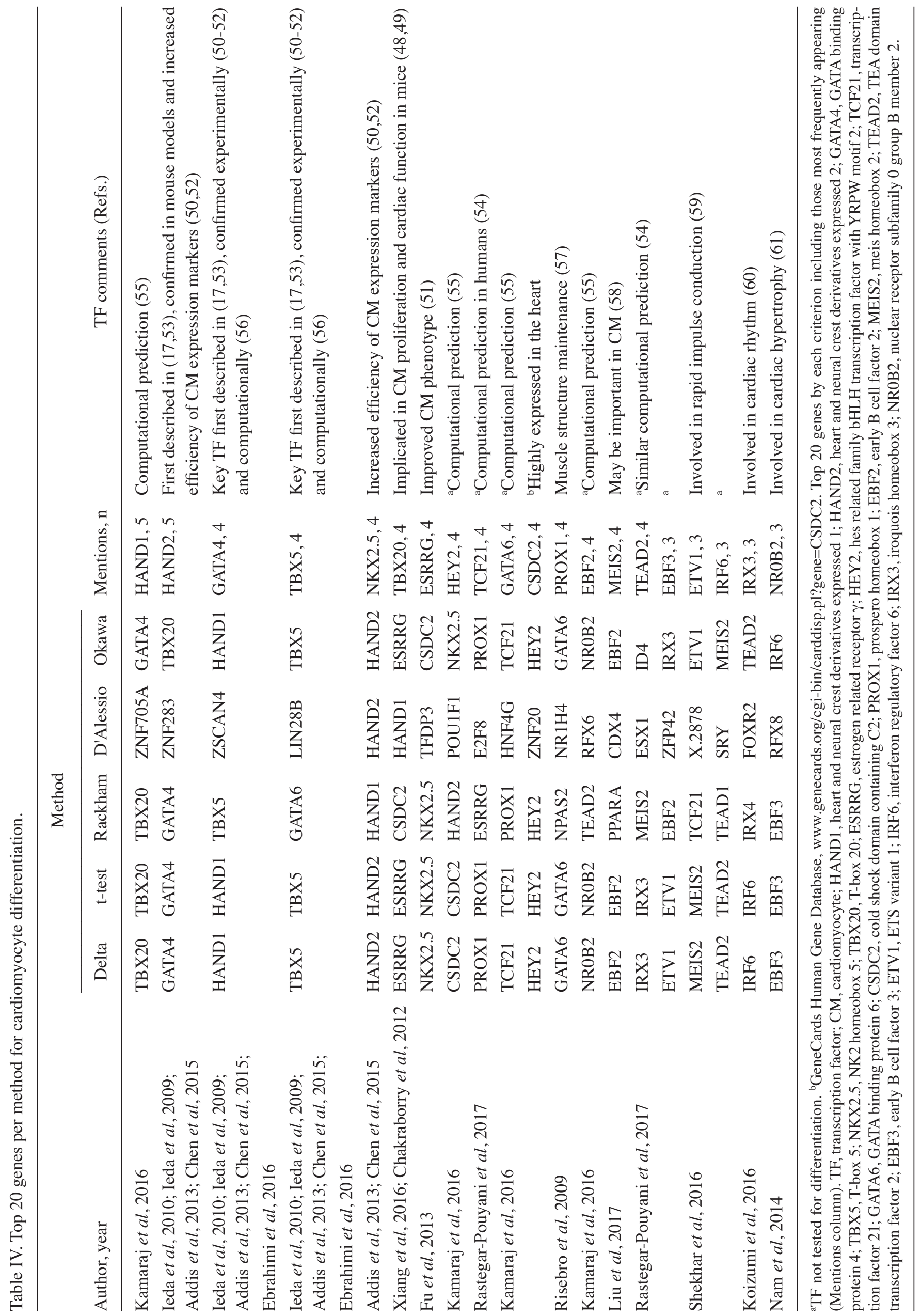


A Delta

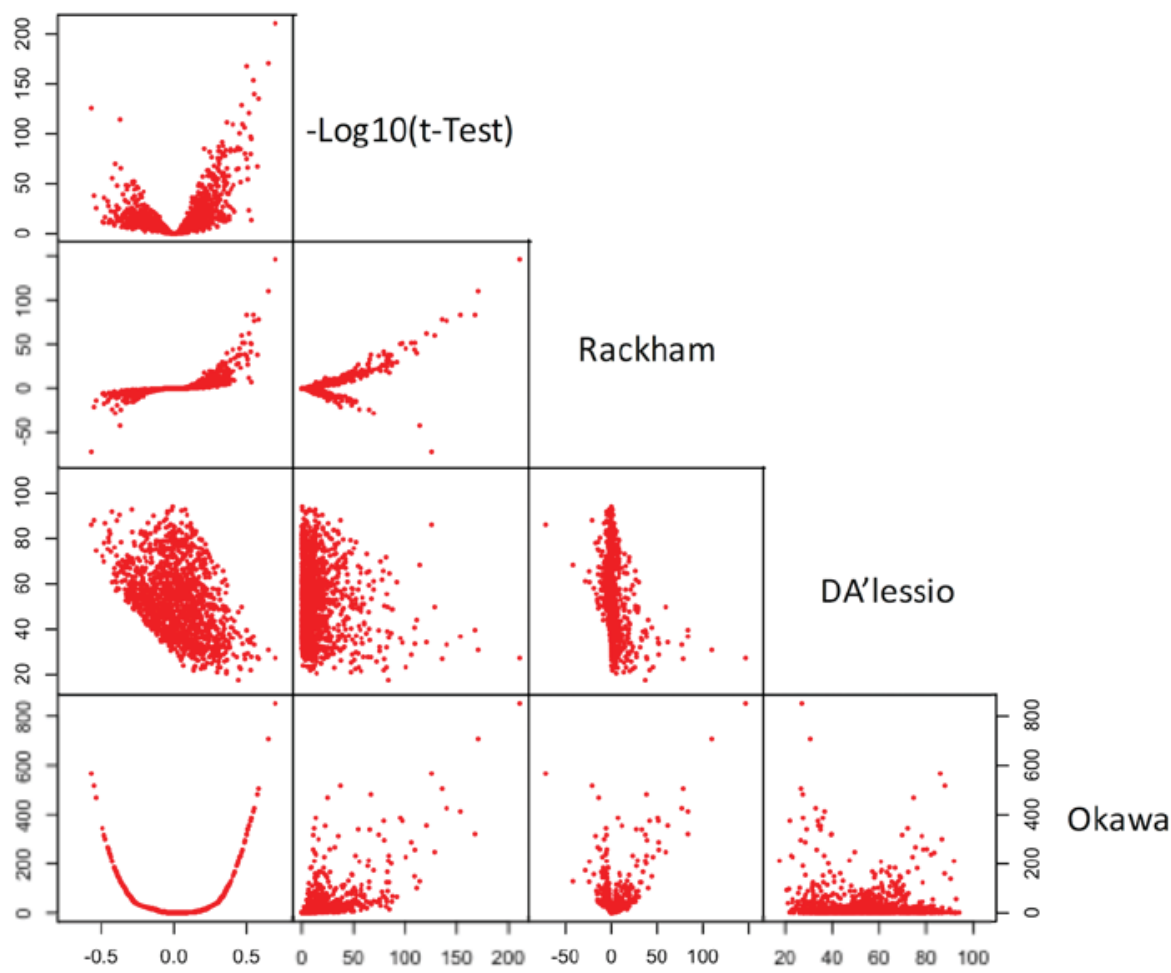

B

\begin{tabular}{ccccc|cc}
\hline Delta & T.Test & Rackham & DA'lessio & Okawa & \multicolumn{2}{c}{ Mentions } \\
\hline POU6F2 & POU6F2 & POU6F2 & TCF24 & POU6F2 & POU6F2 & 4 \\
TFAP2B & TFAP2B & TFAP2B & ZNF674 & TFAP2B & TFAP2B & 4 \\
TSC22D1 & ZNF480 & ZNF460 & DMBX1 & MEIS2 & ZNF480 & 4 \\
LMX1B & ZNF460 & ZNF480 & PRDM8 & IRF8 & ZNF460 & 4 \\
RRN3P1 & RRN3P1 & TSC22D1 & GSC & TSC22D1 & RRN3P1 & 4 \\
ZNF460 & TSC22D1 & RRN3P1 & ZNF836 & LMX1B & TSC22D1 & 4 \\
ZNF208 & NFAT5 & ZFHX4 & ZNF90 & PRDM1 & ZFHX4 & 4 \\
EMX2 & MEIS2 & NFAT5 & FOXR1 & RRN3P1 & GLIS3 & 4 \\
ZNF836 & ZFHX4 & MTA2 & ZNF497 & ZNF460 & EMX2 & 4 \\
GLIS3 & NR1H4 & PRDM5 & ZNF99 & ZNF208 & ZNF836 & 4 \\
ZFHX4 & GTF2A2 & GLIS3 & GSX1 & EMX2 & NFAT5 & 3 \\
ZNF141 & MTA2 & EMX2 & C20orf144 & ZNF836 & MTA2 & 3 \\
RUNX1T1 & F1 1 & GATAD1 & GATAD1 & GLIS3 & PRDM5 & 3 \\
MBD2 & PRDM5 & YY1 & ZNF620 & ZFHX4 & GATAD1 1 & 3 \\
ZNF480 & GATAD1 & ZNF836 & ZNF491 & ZNF141 & LMX1B & 3 \\
ERG & GLIS3 & GTF2A2 & TBX20 & ZNF770 & MEIS2 & 2 \\
ZNF382 & EMX2 & ZNF382 & ZNF560 & RUNX1T1 & GTF2A2 & 2 \\
PRDM5 & ZNF292 & BCLAF1 & LBX1 & MBD2 & YY1 & 2 \\
MTA2 & NR2C1 & LMX1B & GSC2 & ZNF480 & ZNF382 & 2 \\
NFAT5 & BTF3L4 & PITX2 & HOXD4 & ERG & ERG & 2 \\
\hline
\end{tabular}

C

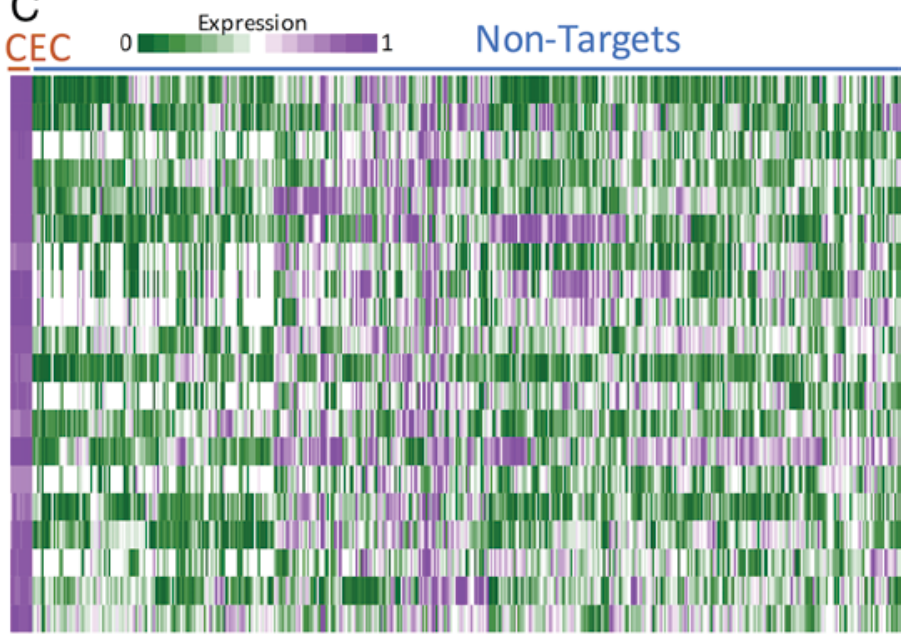

Figure 5. Results for the CEC example. (A) Comparison of the five scores. The t-test P-value is indicated as -Log10. (B) Table of the top 20 genes by each criterion including those most frequently arising (Mentions column). Genes were assigned specific colors. Genes in italics were repeated, although not in the top 20. Black genes were specific to each score. (C) Comparison of gene expression of genes in column Mentions in panel (B) across CEC and non-target cell types. CEC, corneal endothelial cells.

clear that, apart from D'Alessio, the majority of the genes were frequently in the top TFs, irrespective of the score. In Delta and $t$-test, there was no selection for overexpressed genes and therefore some underexpressed TFs appeared, including meis homeobox 2 (MEIS2) and zinc finger protein 208. Similarly, in Okawa, the metric implemented did not favor overexpression in the target and certain genes appeared to be underexpressed, including interferon regulatory factor 8 and MEIS2 (the script available was commented so as to be able to alter this easily). The lack of similarity of the D'Alessio TFs (2 out of 20) reflected the inappropriate implementation or deficiencies in providing details for reproduction.
Specificity of TF expression. Fig. 5C demonstrates the expression of the 20 most frequent TFs, as listed in the column Mentions in Fig. 5B. It is clear that the expression of all TFs was high in CEC. Subsets of these genes, however, exhibited high expression in other cell types. This result suggested a highly specific profile for CEC. Lim homeobox transcription factor $1 \beta$, for instance, is essential for the correct development of the cornea and other eye structures in mice (65), POU class 6 homeobox 2 is required for retinal regeneration in zebrafish (66), transcription factor AP-2 $\beta$ has been demonstrated to control differentiated CEC markers (67), TSC22 domain family member 1 is 
downregulated in dry eye syndrome (68), and GLIS family zinc finger 3 has been associated with glaucoma (69). This small literature analysis suggests that the observed list of TFs is important in CEC. To select more specific TFs, however, it is necessary to perform a network analysis (summarized in Fig. 4), literature revision, comparison of this profile with the source cell type, and analysis of the gene expression levels of these TFs and other differentially expressed genes (non-TF genes).

\section{Conclusions}

In conclusion, there is a requirement for specific cell types in regenerative medicine and biological research. An interesting proposal is the direct conversion of easy-to-obtain cells, which requires a specific cocktail of TFs to induce alterations in the cell state. Despite the complexity of the computational methods proposed for this task, it was demonstrated that the strategies to identify the TFs involved in the molecular state maintenance of a cell type are relatively simple: i) Define cell populations representing diverse cell types; ii) identify differences in TF expression; and iii) apply rules to remove unlikely TFs. The present review reported that the principal complexity in the computational methods is the third of these points. It was demonstrated in a well-known cardiomyocyte example and a novel corneal endothelial cell example that applying the first two easy-to-implement ideas is likely to provide useful results, which may provide important insights and a starting point for laboratory assays. The present review may additionally inspire novel computational methods to identify TFs associated with cell identity and direct cell conversions.

\section{Acknowledgements}

Not applicable.

\section{Funding}

The present study was funded by CONACyT Ciencia Básica (grant no. 255747) and the Grupos de Investigación con Enfoque Estratégico en Bioinformática para el Diagnóstico Clínico from Tecnológico de Monterrey including a scholarship for GIGR and sponsorship of JFIC.

\section{Availability of data and materials}

The datasets generated and/or analyzed during the current study are available from: http://bioinformatica.mty.itesm. $\mathrm{mx} / \mathrm{CEC}$-TF-Example.

\section{Authors' contributions}

GIGR, CMVC, JFIC and VT made analyses of particular methods and participated in the overall conceptualization. GIGR selected and preprocessed the gene expression omnibus data. GIGR, CMVC and JFIC drafted the initial manuscript. VT conceptualized and supervised the study, wrote the $\mathrm{R}$ scripts and performed the computational analyses. GIGR and VT participated in writing the final manuscript. All authors read and approved the final manuscript.

\section{Ethics approval and consent to participate}

Not applicable.

\section{Consent for publication}

Not applicable.

\section{Competing interests}

The authors declare that they have no competing interests.

\section{References}

1. Abdel Meguid E, Ke Y, Ji J and El-Hashash AHK: Stem cells applications in bone and tooth repair and regeneration: New insights, tools and hopes. J Cell Physiol 233: 1825-1835, 2018.

2. Tabar V and Studer L: Pluripotent stem cells in regenerative medicine: Challenges and recent progress. Nat Rev Genet 15: 82-92, 2014.

3. Valdez-Garcia JE, Zavala J and Trevino V: Current state and future perspectives in corneal endothelium differentiation. In: Frontiers in Stem Cell and Regenerative Medicine Research. Atta-ur-Rahman and Anjum S (eds.) Bentham, 2017.

4. Mora C, Serzanti M, Consiglio A, Memo M and Dell'Era P: Clinical potentials of human pluripotent stem cells. Cell Biol Toxicol 33: 351-360, 2017.

5. López-González R and Velasco I: Therapeutic potential of motor neurons differentiated from embryonic stem cells and induced pluripotent stem cells. Arch Med Res 43: 1-10, 2012.

6. Lizio M, Harshbarger J, Shimoji H, Severin J, Kasukawa T, Sahin S, Abugessaisa I, Fukuda S, Hori F, Ishikawa-Kato S, et al: Gateways to the FANTOM5 promoter level mammalian expression atlas. Genome Biol 16: 22, 2015.

7. Li M and Belmonte JC: Ground rules of the pluripotency gene regulatory network. Nat Rev Genet 18: 180-191, 2017.

8. Takahashi K and Yamanaka S: Induction of pluripotent stem cells from mouse embryonic and adult fibroblast cultures by defined factors. Cell 126: 663-676, 2006.

9. Li M, Liu G and Izpisua Belmonte JC: Navigating the epigenetic landscape of pluripotent stem cells. Nat Rev Mol Cell Biol 13: 524-535, 2012.

10. Moris N, Pina C and Arias AM: Transition states and cell fate decisions in epigenetic landscapes. Nat Rev Genet 17: 693-703, 2016.

11. Vaquerizas JM, Kummerfeld SK, Teichmann SA and Luscombe NM: A census of human transcription factors: Function, expression and evolution. Nat Rev Genet 10: 252-263, 2009.

12. Frum $\mathrm{T}$ and Ralston $\mathrm{A}$ : Cell signaling and transcription factors regulating cell fate during formation of the mouse blastocyst. Trends Genet 31: 402-410, 2015.

13. Morris SA: Direct lineage reprogramming via pioneer factors; a detour through developmental gene regulatory networks. Development 143: 2696-2705, 2016.

14. Iwafuchi-Doi M and Zaret KS: Cell fate control by pioneer transcription factors. Development 143: 1833-1837, 2016.

15. Takahashi K, Tanabe K, Ohnuki M, Narita M, Ichisaka T, Tomoda $\mathrm{K}$ and Yamanaka S: Induction of pluripotent stem cells from adult human fibroblasts by defined factors. Cell 131: 861-872, 2007.

16. Vierbuchen T, Ostermeier A, Pang ZP, Kokubu Y, Südhof TC and Wernig M: Direct conversion of fibroblasts to functional neurons by defined factors. Nature 463: 1035-1041, 2010.

17. Ieda M, Fu JD, Delgado-Olguin P, Vedantham V, Hayashi Y, Bruneau BG and Srivastava D: Direct reprogramming of fibroblasts into functional cardiomyocytes by defined factors. Cell 142: 375-386, 2010.

18. Protze S, Khattak S, Poulet C, Lindemann D, Tanaka EM and Ravens U: A new approach to transcription factor screening for reprogramming of fibroblasts to cardiomyocyte-like cells. J Mol Cell Cardiol 53: 323-332, 2012. 
19. Bonilla-Porras AR, Velez-Pardo $\mathrm{C}$ and Jimenez-Del-Rio $\mathrm{M}$ : Fast transdifferentiation of human Wharton's jelly mesenchymal stem cells into neurospheres and nerve-like cells. J Neurosci Methods 282: 52-60, 2017.

20. Abad M, Hashimoto H, Zhou H, Morales MG, Chen B, Bassel-Duby R and Olson EN: Notch inhibition enhances cardiac reprogramming by increasing MEF2C transcriptional activity. Stem Cell Rep 8: 548-560, 2017.

21. Islas JF, Liu Y, Weng KC, Robertson MJ, Zhang S, Prejusa A, Harger J, Tikhomirova D, Chopra M, Iyer D, et al: Transcription factors ETS2 and MESP1 transdifferentiate human dermal fibroblasts into cardiac progenitors. Proc Natl Acad Sci USA 109: 13016-13021, 2012.

22. Waddington $\mathrm{CH}$ : The strategy of the genes. Routledge, 1957.

23. Cahan P, Li H, Morris SA, Lummertz Da Rocha E, Daley GQ and Collins JJ: CellNet: Network biology applied to stem cell engineering. Cell 158: 903-915, 2014.

24. D'Alessio AC, Fan ZP, Wert KJ, Baranov P, Cohen MA, Saini JS, Cohick E, Charniga C, Dadon D, Hannett NM, et al: A systematic approach to identify candidate transcription factors that control cell identity. Stem Cell Reports 5: 763-775, 2015.

25. Rackham OJL, Firas J, Fang H, Oates ME, Holmes ML, Knaupp AS; FANTOM Consortium, Suzuki H, Nefzger CM, Daub CO, et al: A predictive computational framework for direct reprogramming between human cell types. Nat Genet 48: 331-335, 2016.

26. Okawa S, Nicklas S, Zickenrott S, Schwamborn JC and Del Sol A: A generalized gene-regulatory network model of stem cell differentiation for predicting lineage specifiers. Stem Cell Rep 7: 307-315, 2016

27. Barrett T, Suzek TO, Troup DB, Wilhite SE, Ngau WC, Ledoux P, Rudnev D, Lash AE, Fujibuchi W and Edgar R: NCBI GEO: Mining millions of expression profiles-database and tools. Nucleic Acids Res 33(Database Issue): D562-D566, 2005.

28. Barrett T, Wilhite SE, Ledoux P, Evangelista C, Kim IF, Tomashevsky M, Marshall KA, Phillippy KH, Sherman PM, Holko M, et al: NCBI GEO: Archive for functional genomics data sets-update. Nucleic Acids Res 41 (Database Issue): D991-D995, 2013.

29. Parkinson H, Kapushesky M, Shojatalab M, Abeygunawardena $N$ Coulson R, Farne A, Holloway E, Kolesnykov N, Lilja P, Lukk M, et al: ArrayExpress-a public database of microarray experiments and gene expression profiles. Nucleic Acids Res 35 (Database Issue): D747-D750, 2007.

30. Kolesnikov N, Hastings E, Keays M, Melnichuk O, Tang YA, Williams E, Dylag M, Kurbatova N, Brandizi M, Burdett T, et al: ArrayExpress update-simplifying data submissions. Nucleic Acids Res 43 (Database Issue): D1113-D1116, 2015.

31. Rosenbloom KR, Sloan CA, Malladi VS, Dreszer TR, Learned K, Kirkup VM, Wong MC, Maddren M, Fang R, Heitner SG, et al: ENCODE Data in the UCSC genome browser: Year 5 update. Nucleic Acids Res 41 (Database Issue): D56-D63, 2013.

32. Kim SY, Lee JW and Sohn IS: Comparison of various statistical methods for identifying differential gene expression in replicated microarray data. Stat Methods Med Res 15: 3-20, 2006

33. Huang HC, Niu Y and Qin LX: Differential expression analysis for RNA-Seq: An overview of statistical methods and computational software. Cancer Inform 14 (Suppl 1): S57-S67, 2015.

34. Seyednasrollah F, Laiho A and Elo LL: Comparison of software packages for detecting differential expression in RNA-seq studies. Brief Bioinform 16: 59-70, 2013.

35. Sullivan GM and Feinn R: Using effect size-or why the $P$ value is not enough. J Grad Med Educ 4: 279-282, 2012.

36. Huang S, Guo YP, May G and Enver T: Bifurcation dynamics in lineage-commitment in bipotent progenitor cells. Dev Biol 305 695-713, 2007.

37. Jacob F and Monod J: Genetic regulatory mechanisms in the synthesis of proteins. J Mol Biol 3: 318-356, 1961.

38. Roeder I and Glauche I: Towards an understanding of lineage specification in hematopoietic stem cells: A mathematical mode for the interaction of transcription factors GATA-1 and PU.1. J Theor Biol 241: 852-865, 2006

39. Faith JJ, Hayete B, Thaden JT, Mogno I, Wierzbowski J, Cottarel G, Kasif S, Collins JJ and Gardner TS: Large-scale mapping and validation of escherichia coli transcriptional regulation from a compendium of expression profiles. PLoS Biol 5: e8, 2007.

40. Rosvall $\mathrm{M}$ and Bergstrom CT: Maps of random walks on complex networks reveal community structure. Proc Natl Acad Sci USA 105: 1118-1123, 2008.
41. Subramanian A, Tamayo P, Mootha VK, Mukherjee S, Ebert BL, Gillette MA, Paulovich A, Pomeroy SL, Golub TR, Lander ES and Mesirov JP: Gene set enrichment analysis: A knowledge-based approach for interpreting genome-wide expression profiles. Proc Natl Acad Sci USA 102: 15545-15550, 2005.

42. FANTOM Consortium H, Suzuki H, Forrest AR, van Nimwegen E, Daub CO, Balwierz PJ, Irvine KM, Lassmann T, Ravasi T, Hasegawa Y, et al: The transcriptional network that controls growth arrest and differentiation in a human myeloid leukemia cell line. Nat Genet 41: 553-562, 2009.

43. Szklarczyk D, Franceschini A, Wyder S, Forslund K, Heller D, Huerta-Cepas J, Simonovic M, Roth A, Santos A, Tsafou KP, et al: STRING v10: Protein-protein interaction networks, integrated over the tree of life. Nucleic Acids Res 43 (Database Issue): D447-D452, 2015.

44. Nikolsky Y, Ekins S, Nikolskaya T and Bugrim A: A novel method for generation of signature networks as biomarkers from complex high throughput data. Toxicol Lett 158: 20-29, 2005.

45. Crespo I and Del Sol A: A general strategy for cellular reprogramming: The importance of transcription factor cross-repression. Stem Cells 31: 2127-2135, 2013.

46. Kauffman SA: Homeostasis and differentiation in random genetic control networks. Nature 224: 177-178, 1969.

47. Zhang HM, Liu T, Liu CJ, Song S, Zhang X, Liu W, Jia H, Xue Y and Guo AY: AnimalTFDB 2.0: A resource for expression, prediction and functional study of animal transcription factors. Nucleic Acids Res 43(Database Issue): D76-D81, 2015.

48. Xiang FL, Guo M and Yutzey KE: Overexpression of Tbx20 in adult cardiomyocytes promotes proliferation and improves cardiac function after myocardial infarction. Circulation 133: 1081-1092, 2016

49. Chakraborty S and Yutzey KE: Tbx20 regulation of cardiac cell proliferation and lineage specialization during embryonic and fetal development in vivo. Dev Biol 363: 234-246, 2012.

50. Addis RC, If kovits JL, Pinto F, Kellam LD, Esteso P, Rentschler S, Christoforou N, Epstein JA and Gearhart JD: Optimization of direct fibroblast reprogramming to cardiomyocytes using calcium activity as a functional measure of success. J Mol Cell Cardiol 60: 97-106, 2013.

51. Fu JD, Stone NR, Liu L, Spencer CI, Qian L, Hayashi Y, Delgado-Olguin P, Ding S, Bruneau BG and Srivastava D: Direct reprogramming of human fibroblasts toward a cardiomyocyte-like state. Stem Cell Reports 1: 235-247, 2013.

52. Chen $\mathrm{O}$ and Qian L: Direct cardiac reprogramming: Advances in cardiac regeneration. Biomed Res Int 2015: 580406, 2015.

53. Ieda M, Tsuchihashi T, Ivey KN, Ross RS, Hong TT, Shaw RM and Srivastava D: Cardiac fibroblasts regulate myocardial proliferation through beta1 integrin signaling. Dev Cell 16: 233-244, 2009.

54. Rastegar-Pouyani S, Khazaei N, Wee P, Yaqubi M and Mohammadnia A: Meta-analysis of transcriptome regulation during induction to cardiac myocyte fate from mouse and human fibroblasts. J Cell Physiol 232: 2053-2062, 2017.

55. Kamaraj US, Gough J, Polo JM, Petretto E and Rackham OJ: Computational methods for direct cell conversion. Cell Cycle 15: 3343-3354, 2016.

56. Ebrahimi B: Biological computational approaches: New hopes to improve (re)programming robustness, regenerative medicine and cancer therapeutics. Differentiation 92: 35-40, 2016.

57. Risebro CA, Searles RG, Melville AA, Ehler E, Jina N, Shah S, Pallas J, Hubank M, Dillard M, Harvey NL, et al: Prox1 maintains muscle structure and growth in the developing heart. Development 136: 495-505, 2009.

58. Liu Q, Jiang C, Xu J, Zhao MT, Van Bortle K, Cheng X, Wang G, Chang HY, Wu JC and Snyder MP: Genome-wide temporal profiling of transcriptome and open chromatin of early cardiomyocyte differentiation derived from hiPSCs and hESCs. Circ Res 121: 376-391, 2017

59. Shekhar A, Lin X, Liu FY, Zhang J, Mo H, Bastarache L, Denny JC, Cox NJ, Delmar M, Roden DM, et al: Transcription factor ETV1 is essential for rapid conduction in the heart. J Clin Invest 126: 4444-4459, 2016.

60. Koizumi A,Sasano T, Kimura W, Miyamoto Y,Aiba T, Ishikawa T, Nogami A, Fukamizu S, Sakurada H, Takahashi Y, et al: Genetic defects in a His-Purkinje system transcription factor, IRX3, cause lethal cardiac arrhythmias. Eur Heart J 37: 1469-1475, 2016.

61. Nam YS, Kim Y, Joung H, Kwon DH, Choe N, Min HK, Kim YS, Kim HS, Kim DK, Cho YK, et al: Small heterodimer partner blocks cardiac hypertrophy by interfering with GATA6 signaling. Circ Res 115: 493-503, 2014. 
62. Frausto RF, Wang C and Aldave AJ: Transcriptome analysis of the human corneal endothelium. Invest Ophthalmol Vis Sci 55: 7821-7830, 2014

63. Trevino V: Chi-Co-Express: A database of human co-expression networks from global cell states. Manuscr Prep, 2017.

64. Li Q, Birkbak NJ, Gyorffy B, Szallasi Z and Eklund AC: Jetset: Selecting the optimal microarray probe set to represent a gene. BMC Bioinformatics 12: 474, 2011.

65. Pressman CL, Chen H and Johnson RL: LMX1B, a LIM homeodomain class transcription factor, is necessary for normal development of multiple tissues in the anterior segment of the murine eye. Genesis 26: 15-25, 2000.

66. Powell C, Cornblath E and Goldman D: Zinc-binding domain-dependent, deaminase-independent actions of apolipoprotein B mRNA-editing enzyme, catalytic polypeptide 2 (Apobec2), mediate its effect on zebrafish retina regeneration. J Biol Chem 289: 28924-28941, 2014.
67. Chen L, Martino V, Dombkowski A, Williams T, West-Mays J and Gage PJ: AP-2 $\beta$ is a downstream effector of PITX2 required to specify endothelium and establish angiogenic privilege during corneal development. Invest Opthalmol Vis Sci 57: 1072-1081, 2016.

68. Bradley JL, Edwards CS and Fullard RJ: Adaptation of impression cytology to enable conjunctival surface cell transcriptome analysis. Curr Eye Res 39: 31-41, 2014.

69. Khor CC, Do T, Jia H, Nakano M, George R, Abu-Amero K, Duvesh R, Chen LJ, Li Z, Nongpiur ME, et al: Genome-wide association study identifies five new susceptibility loci for primary angle closure glaucoma. Nat Genet 48: 556-562, 2016. 